\title{
Maternal and Perinatal outcome of Eclampsia at a third level Tunisian hospital: a 12 years review
}

\author{
F. Ferhi, K. Tarmiz, A. Khlifi*, M. A. Bouslama, H. KHOUADJA, K. Benjazia \\ Department of Anesthesiology and Critical Care Medicine. Farhat Hached University Teaching Hospital. Sousse Tunisia \\ * Department of Obstetrics and Gynecology. Farhat Hached University Teaching Hospital. Sousse 4002. Tunisia
}

\section{BACKGROUND AND GOAL OF STUDY:}

Despite advances in detection and management, preeclampsia/eclampsia remains a common cause of maternal morbidity and death in the world.

The aim of this study was to assess the incidence of eclampsia and its morbidity and mortality.

\section{MATERIALS AND METHODS:}

We conducted a retrospective survey in a third level Tunisian university teaching hospital from January 2004 to December 2016.

We included all patients with the diagnosis of eclampsia. We evaluated clinical profile, maternal and perinatal outcome.

\section{RESULTS:}

In our study period, 129847 deliveries were registered. Eighty-three women with eclampsia were identified. Hence, the incidence of eclampsia was 64 per 100000 deliveries.

There was a bimodal distribution in age with the highest frequency at 21 and 41 years.

All cases of eclampsia occurred before delivery. A quarter of first seizures occurred in hospital.
The median gestational age at the time of eclampsia was 35 weeks of gestation.

The delivery mode was caesarean section in $100 \%$.

The recurrence of eclampsia despite magnesium sulfate prevention was observed in $6 \%$ of patients.

Severe complications of eclampsia were recorded in $10.8 \%$ of patients:

- Four cases of posterior reversible encephalopathy syndrome,

- Two cases of acute pulmonary edema,

- and 3 cases of HELLP syndrome.

No maternal deaths due to eclampsia were recorded.

Only $10.8 \%$ of new born were preterm. There were 5 stillbirths and 2 neonatal deaths.

\section{CONCLUSION:}

The incidence of eclampsia was very high probably due to center effect. It's essential to raise awareness among mothers in the community regarding early signs and symptoms

of preeclampsia / eclampsia and to design a better tracking system for antenatal care program. 